\title{
Evaluation of the fetal QT interval using non-invasive
}

\section{fetal ECG technology}

Joachim Behar ${ }^{1,2}$, Tingting Zhu², Julien Oster ${ }^{2}$, Alisa Niksch ${ }^{3}$, Douglas Y. Mah ${ }^{4}$, Terrence Chun ${ }^{5}$, James Greenberg ${ }^{6}$, Cassandre Tanner ${ }^{7}$, Jessica Harrop ${ }^{6}$, Reza Sameni ${ }^{8}$, Jay Ward ${ }^{9}$, Adam J.

Wolfberg 9 , Gari D. Clifford ${ }^{9,10}$

1. Biomedical Engineering Faculty, Technion-IIT, Haifa, Israel; 2. University of Oxford, Oxford, UK; 3. Department of Pediatric Cardiology, Tufts Medical Center, Boston, MA; 4. Department of Pediatric Cardiology, Boston Children's Hospital, Boston, MA; 5. Department of Pediatric Cardiology, Seattle Children's Hospital, Seattle, WA; 6. Department of OBGYN, Brigham and Women's Hospital, Boston, MA; 7. Department of OBGYN, Tufts Medical Center, Boston, MA; 8. Shiraz University, Iran; 9. Mindchild Medical, North Andover, MA; 10. Departments of Biomedical Informatics and Biomedical Engineering, Emory University and, Georgia Institute of Technology, Atlanta, GA

E-mail: gari@gatech.edu 


\begin{abstract}
Non-invasive fetal electrocardiography (NI-FECG) is a promising alternative continuous fetal monitoring method that has the potential to allow morphological analysis of the FECG. However, there are a number of challenges associated with the evaluation of morphological parameters from the NIFECG, including low signal to noise ratio of the NI-FECG and methodological challenges for getting reference annotations and evaluating the accuracy of segmentation algorithms. This work aims to validate the measurement of the fetal QT interval in term laboring women using a NI-FECG electrocardiogram monitor. Fetal electrocardiogram data were recorded from 22 laboring women at term using the NI-FECG and an invasive fetal scalp electrode simultaneously. A total of 105 one-minute epochs were selected for analysis. Three pediatric electrophysiologists independently annotated individual waveforms and averaged waveforms from each epoch. The intervals measured on the averaged cycles taken from the NI-FECG and the fetal scalp electrode showed a close agreement; the root mean square error between all corresponding averaged NI-FECG and fetal scalp electrode beats was 13.6 milliseconds, which is lower than the lowest adult root mean square error of 16.1 milliseconds observed in related adult QT studies. These results provide evidence that NI-FECG technology enables accurate extraction of the fetal QT interval.
\end{abstract}

Keywords: non-invasive FECG, ECG morphological analysis, crowd-sourcing, medical annotations. 
Non-invasive fetal QT interval

\section{Introduction}

Continuous fetal heart rate monitoring is the standard of care for intrapartum management in the United States and in many other countries (American College of Obstetricians and Gynecologists, 2005). The limitations of this technology - particularly the very low specificity - are well known, along with the association between the use of continuous fetal heart rate monitoring and an increase in operative vaginal deliveries and cesareans (American College of Obstetricians and Gynecologists, 2005). Obstetricians, however, have few alternatives due to the difficulty in monitoring other physiologic signals from the fetus during pregnancy and labor.

The one fetal signal that has generated the most interest is the fetal ECG waveform, which can be reliably obtained during labor with the use of an invasive fetal scalp electrode (FSE), and less reliably using non-invasive adhesive electrodes attached to the maternal abdomen (Sameni and Clifford, 2010, Wolfberg and Norwitz, 2009, Behar et al., 2016).

Most studied is the ratio between the T-wave and the R-wave, a metric analyzed and reported by the STAN monitor (Neoventa Medical, Goteborg, Sweden) as a proxy for the ST segment. There is a reasonable physiologic basis for monitoring the ST segment during labor as a marker for hypoxia or ischemia (Greene, 1987, Greene and Rosen, 1989). Although a large American study failed to find improved newborn outcomes or reduced cesarean rates when the STAN monitor was used (Belfort et al., 2015), multiple independent trials in Europe have demonstrated significant improvements in newborn outcome when the STAN monitor was used (Amer-Wahlin et al., 2001, Doret et al., 2011, Kessler et al., 2013).

Less research has been conducted on the association between the fetal QT interval and newborn outcome, even though many studies link QT-interval abnormalities during the fetal and newborn period with serious events, including sudden infant death syndrome (Crotti et al., 2013). Oudijk and colleagues used the STAN monitor to measure the QT interval and demonstrated that during severe intrapartum hypoxia and metabolic acidosis, there was a significant shortening of the QT and corrected QT interval (Oudijk et al., 2004). More recently one group identified a fetus as having long QT syndrome using QT measurement performed on the non-invasive fetal ECG (NI-FECG) (Fujimoto et al., 2009). In adults, the QT interval has been of high interest in a number of conditions including the Romano-Ward and Jervell-Lange-Neilson syndromes, drug toxicity, and to predict prognosis following acute myocardial infarction (Campbell et al., 1985).

Other pathologic conditions linked to an abnormal QT interval include an association between a prolonged QT interval in newborns and the use of selective serotonin reuptake inhibitors (SSRI) during 
Non-invasive fetal QT interval

pregnancy (Dubnov et al., 2005, Dubnov-Raz et al., 2008). These observations suggest the potential to screen for adverse events using the fetal QT interval during pregnancy and labor.

Hampering research is the requirement that a wire electrode be directly attached to the fetal scalp in order to obtain a reliable signal. Placement of the FSE requires ruptured membranes and a dilated cervix and thus this modality is limited to monitoring during labor. Furthermore, the FSE does not allow for monitoring of the fetus prior to labor, and because the FSE has only one electrode on the fetal scalp, it does not cover the three dimensional electrical field emanating from the fetal heart. In contrast, the NI-FECG monitor could be used for antepartum (as well as intrapartum) fetal monitoring and it provides a three dimensional electrical representation of the electrical field emanating from the fetal heart. Thus, there is a strong motivation for developing a non-invasive method for measuring the FECG obtained from multiple abdominal ECG sensors. Indeed, NI-FECG is a non-invasive monitoring method that allows to estimate the FHR, as well as information on the electrical activity of the heart which is embedded in the ECG morphology.

Accurate extraction of the FHR from the NI-FECG has been demonstrated (Behar et al., 2014, Clifford et al., 2014). Our group previously has described the accurate measurement of the ST segment from the external fetal ECG recordings (Clifford et al., 2011). However, accurate QT interval estimation from NI-FECG has not been previously demonstrated.

To be clinically useful, the fetal QT interval measured using abdominal ECG technology must be reliably identical to the fetal QT interval measured using a direct ECG measurement. We sought to validate the non-invasive measurement of the fetal QT interval in order to allow for additional research to be conducted without the need for a FSE. This paper describes the method for rigorously comparing the fetal QT intervals extracted from the NI-FECG and FSE, and demonstrates the feasibility of fetal QT measurement from the NIFECG signal.

\section{Materials and methods}

The study was approved by the Institutional Review Boards at the institutions where data were collected: Brigham and Women's: 2010-P-00278/1, Cleveland Clinic Fairview: 12-154, Newton Wellesley: N08-445 and Tufts Medical Center: 7863 20. Fetal ECG data were recorded from 22 term laboring women with singleton fetuses. Data were recorded simultaneously using a 28 NI-FECG monitor (Mindchild Medical, North Andover, MA) and a single lead invasive FSE (GE Corometrics). Data were recorded at a sampling 
Non-invasive fetal QT interval

frequency of $1 \mathrm{kHz}$ with 16-bit accuracy. All women delivered newborns with five-minute Apgar scores above six, and none of the fetuses were exposed to SSRI medication in-utero. There were no prolonged QT intervals noted by the three independent reviewers of the data or any indications for long QT syndrome from the clinical data.

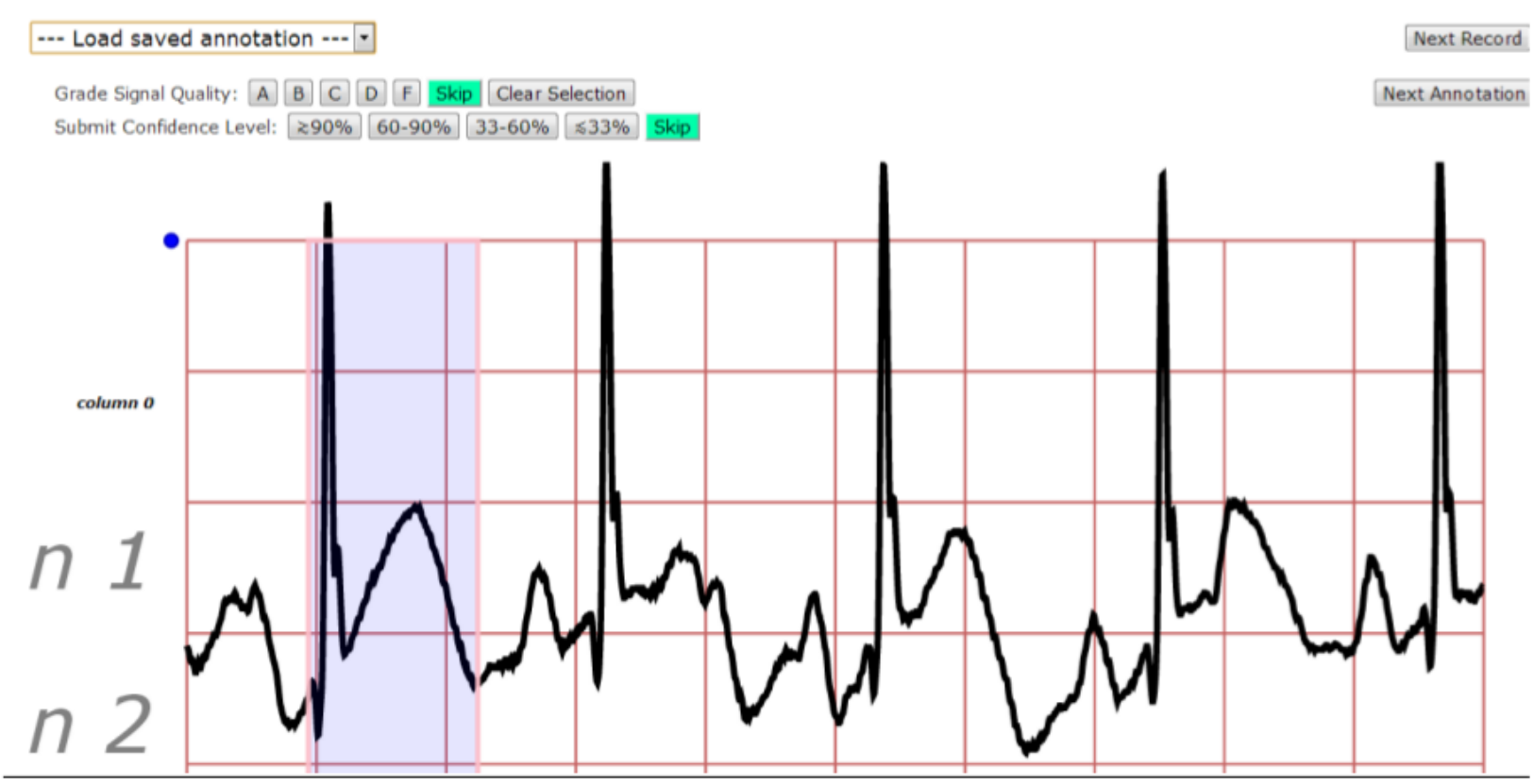

Figure 1: Annotation interface. A fetal QT interval was annotated by dragging a mouse across the interface from left to right (shaded area). The signal in this illustration is a FSE segment. The procedure was also repeated for the ECG derived from the non-invasive FECG.

Each recording period was divided into one-minute segments for the analysis. Segments were selected that had a relatively stable fetal heart rate based on determination that the baseline heart rate did not change by more than $20 \mathrm{bpm}$ during the one-minute period (Silva et al., 2013). In segments that contained accelerations or decelerations (defined as changes from baseline of more than $20 \mathrm{bpm}$ lasting more than 15 seconds) the corresponding sub-segments (generally lasting between 10-15 seconds) were replaced by random noise to ensure the annotators were not annotating in areas with large changes in heart rate. This procedure was implemented to ensure that the fetal QT interval was approximately stable over each one-minute segment, which is necessary when computing averages of ECG cycles (Christov and Simova, 2006). Indeed, a relationship between the QT length and the heart rate has been established in adults (Bazett, 1920) and although such a relationship has not been studied in fetuses, it is reasonable to assume that the QT length be modulated by the fetal heart rate (even if differently than for adults). 
Non-invasive fetal QT interval

The QT interval is defined as the time interval between the Q wave onset and the end of the T wave in the heart's electrical cycle. Three pediatric cardiologists independently annotated the data using the modified Physionet Lightwave interface (Zhu et al., 2014) (example in figure 1). This online system either presented the cardiologists with a rhythm strip one minute long, or presented them with a single ECG waveform created by automatically averaging a series of ECG waveforms. Each cardiologist was trained on the interface individually during an online training session. The precision of the annotation interface was $1 \mathrm{~ms}$.
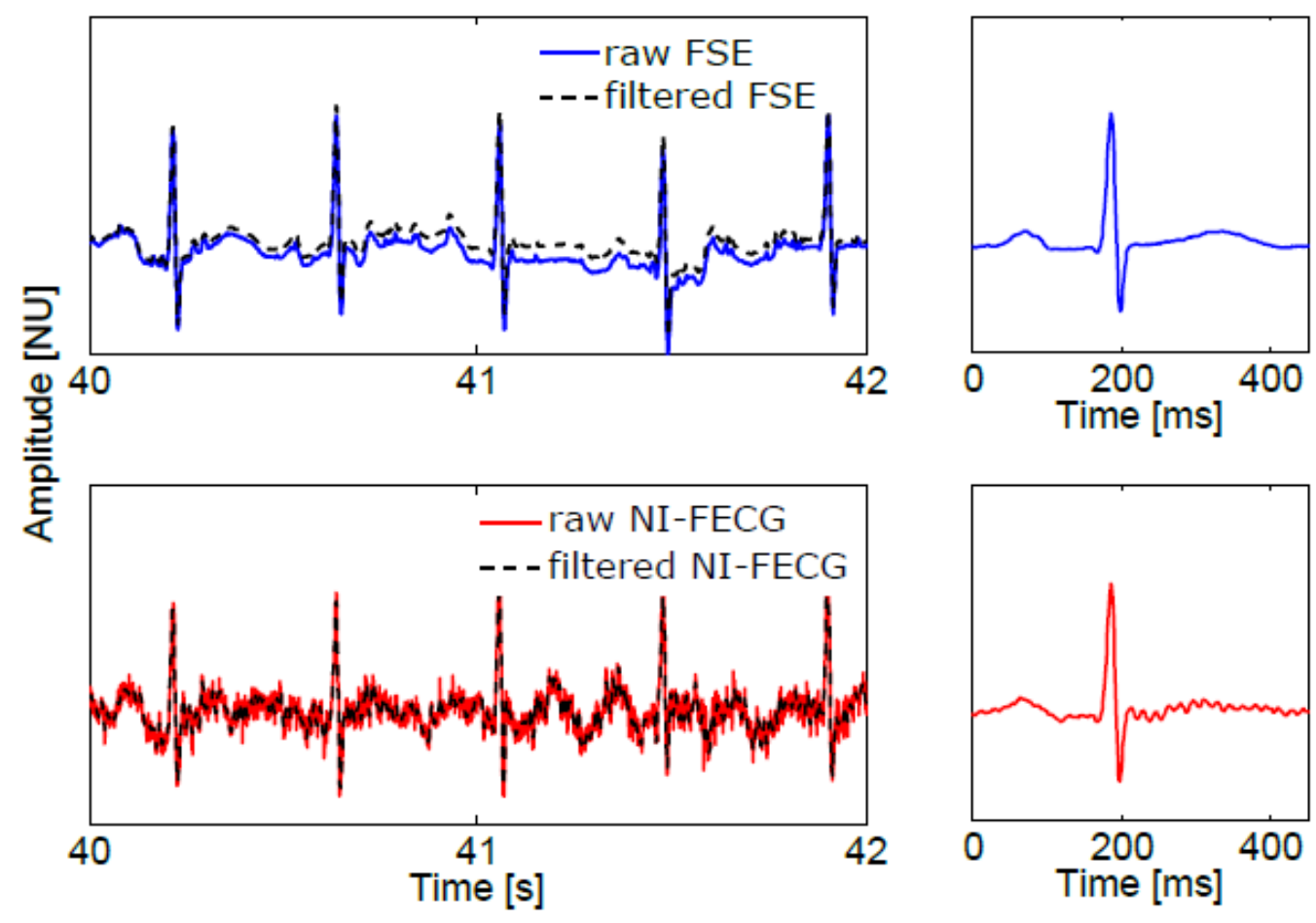

Figure 2: Example of signal used for SET1 and SET2. Top left: raw (solid line) and filtered (dashed) FECG from FSE; Top right: corresponding average ECG; Bottom left: raw (solid line) and filtered (dashed) abdominal NI-ECG; Right: corresponding average ECG templates constructed from the raw ECG signal.

The first set of annotations, denoted SET1, contained 210 one-minute segments (105 recorded using abdominal electrodes and the corresponding 105 segments recorded using a FSE). Each cardiologist was instructed to annotate five QT intervals per one-minute segment, and was told that they were free to choose the five cycles to annotate, a methodology similar to prior manual QT annotation exercises (Moody et al., 2006). The next set, denoted SET2, included 210 averaged fetal ECG cycles (105 abdominal and 105 corresponding FSE). One annotation per waveform was requested. Cardiologists were blinded to signal source (i.e. whether the signal to annotate was FSE or NI-FECG) and the waveforms were presented at random. 
Non-invasive fetal QT interval

In each set, the data were randomized so that two consecutive waveforms were not extracted from the same patient. An example of an annotation made on a rhythm strip segment is demonstrated in figure 1. Examples of signals used in SET1-2 are shown in figure 2.

We analyzed the variation between paired measurements of the QT segment (measured on the NIFECG and FSE). For that purpose the root meant square error (RMSE) and absolute error (AE) were computed. We also evaluated the RMSE95 and AE95 defined as the RMSE and AE evaluated while excluding the extreme $5 \%$ values. This was done to make sure that no outliers in the sample size were biasing the estimation of the RMSE and AE. Three methods for fusing the annotations were investigated: mean, median and an expectation maximization (EM) algorithm (Zhu et al., 2014) - see description in the following paragraph. In addition Wilcoxon signed rank test was applied to test the hypothesis that the difference between scalp and abdominal annotations were samples from continuous distributions with zero median for both SET1 and SET2. The EM algorithm used for fusing the annotations is described in the context of QT annotation in (Zhu et al., 2014). It is assumed that $R$ annotators have annotated a series of $N$, QT observations. The true QT annotation for each individual record is written $z_{i}, i \in[1 ; N]$ and the annotation from annotator $j$ and which was performed on record $i$ is denoted $y_{i}^{j}$. In addition, it is assumed that $z_{i}$ can be predicted using a linear regression model: $z_{i}=$ $\underline{w}^{T} \cdot \underline{x}_{i}+\epsilon$, where $\underline{w}$ is the regression vector and $\epsilon$ is a zero-mean Gaussian noise with precision $\gamma$ and $\underline{x}$ is a feature vector. No features were used in the approach detailed here and thus $\underline{x}$ is a unity vector. The EM algorithm can be summarized as follows:

(1) E-step: the E-step estimates the expected true annotations for all records, $\underline{\hat{z}}$, as a weighted sum of the provided annotations with their precision $\lambda^{j}$. 
Non-invasive fetal QT interval

$$
\underline{\hat{z}}=\frac{\sum_{j=1}^{R} \lambda^{j} \cdot \underline{y}^{j}}{\sum_{j=1}^{R} \lambda^{j}}
$$

(2) The M-step is based on the current estimate of $\underline{\underline{\hat{z}}}$ and given the dataset written $\boldsymbol{D}$. The model parameters such as the regression coefficient $\mathrm{w}$ and precision lambda can be updated using the following equations:

$$
\begin{gathered}
\frac{1}{\widehat{\lambda^{J}}}=\frac{1}{N} \sum_{i=1}^{N}\left(y_{i}^{j}-\underline{\underline{\boldsymbol{w}}}^{T} \cdot \underline{\boldsymbol{x}}_{i}\right)^{2} \\
\underline{\widehat{\underline{w}}}=\left(\sum_{i=1}^{N} \underline{\boldsymbol{x}}_{i} \cdot \underline{\boldsymbol{x}}_{i}^{T}\right)^{-1} \sum_{i=1}^{N} \underline{x}_{i} \cdot \hat{z}_{i}
\end{gathered}
$$

With

$$
\hat{z}_{i}=\frac{\sum_{j=1}^{R} y_{i}^{j} \cdot \lambda^{j}}{\sum_{j=1}^{R} \lambda^{j}}
$$

The precision is initialized as being equal for all annotators (i.e. at the initial step of the algorithm). The initial precision can thus be written as: $\underline{\hat{z}}=\frac{1}{R} \sum_{j=1}^{R} \underline{y}^{j}$. 
Non-invasive fetal QT interval

\section{Results}

Cycles with high correlation were retained to build the averaged cycles and a minimum of 20 cycles per 1 min segment were required to form a valid template. The QT interval measured using the FSE was 0.3 ms shorter, on average, than the QT interval measured using NI-FECG when averaged cycles were annotated and $8.7 \mathrm{~ms}$ longer when individual cycles were annotated. Figure 3 shows the probability density function for the fetal QT interval annotated by the three annotators for SET1 and SET2. On this plot, NI-FECG QT refers to the QT annotated on the NI-FECG extracted using the MindChild monitor and FSE QT refers to the QT annotated on the FSE by the reviewers. For SET2, the two distributions (NI-FECG QT and FSE QT) superimpose almost perfectly (without a significant difference between the distributions), while the NI-FECG QT distribution has a lower median and is more platykurtic (broader) for SET1. For SET1, the null hypothesis of the Wilcoxon signed rank test was rejected under the 5\% significance level whereas the null hypothesis could not be rejected for SET2. This statistical test confirms that the distributions for scalp and abdominal annotations only matched (i.e. were not significantly different) when using the averaged cycles of the NIFECG and FSE.

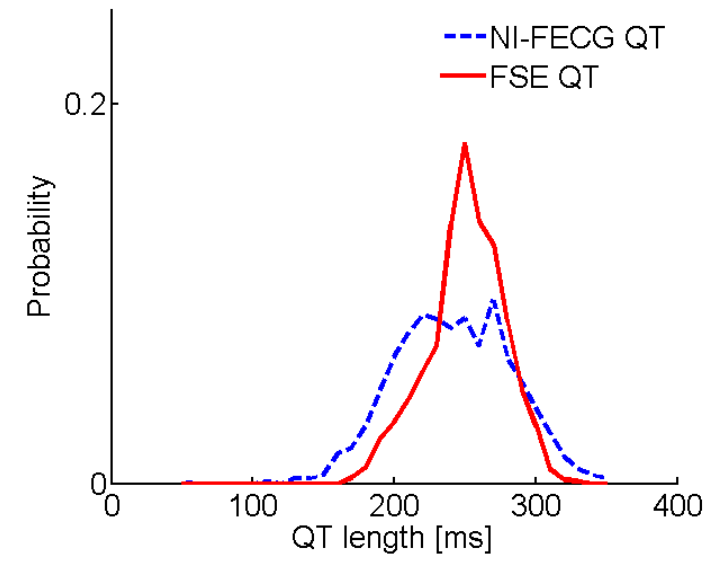

(a)

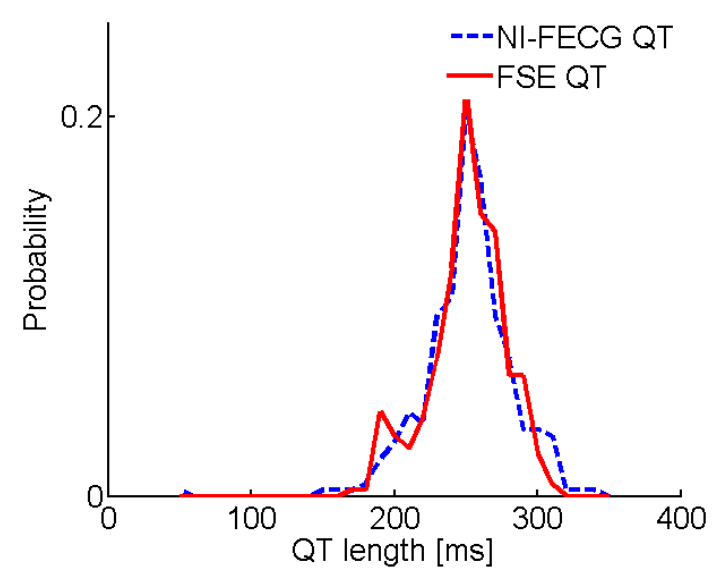

(b)

Figure 3: Empirical probability density function for the median FSE QT interval annotated by the three annotators for: (a) SET1 (i.e. annotation on the raw signals), 3150 annotations, and (b) SET2 (annotation on the averaged heart beat cycles) 630 annotations. For SET2, the two distributions (NI-FECG QT and FSE QT) superimpose closely, while the NI-FECG QT distribution has a lower median and is more platykurtic (broader) than the FSE QT distributions for SET1, indicating more extreme values. 
Non-invasive fetal QT interval

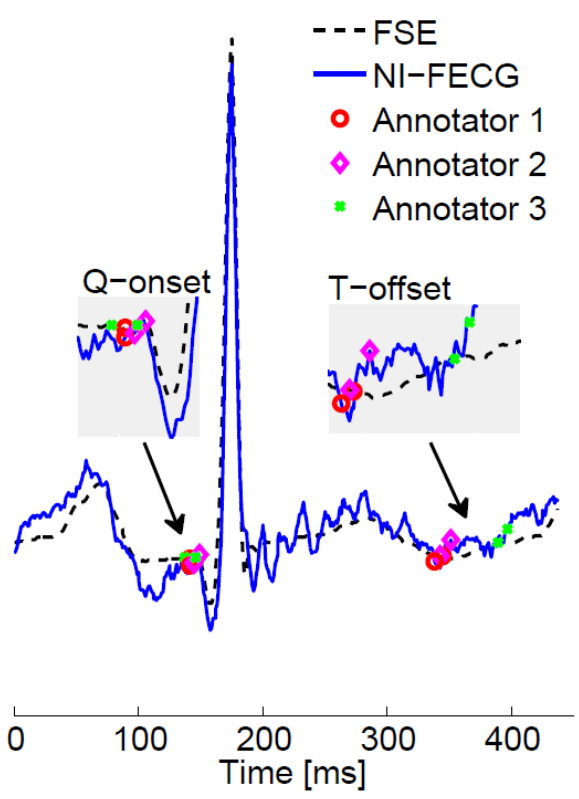

(a)

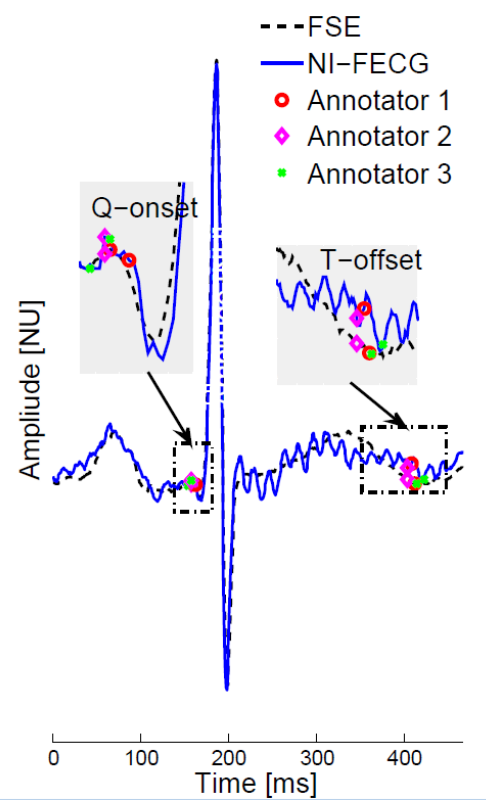

(b)

Figure 4: Comparison of annotations performed on average FECG waveforms from both the FSE and the NIFECG monitor by three experts. (a): note the close correspondence between experts on both the FSE and NIFECG signal. (b): note the disagreement between annotator 3 and the other two experts. This last example illustrates the importance of combining annotators to improve the reliability of results.

Figure 4 shows two examples of averaged cycles (FSE and NI-FECG) being annotated by the three experts. The figure shows the close agreement between the expert annotations on the FSE and on the NI-FECG. A total of 3150 annotations were performed for SET1 (1050 per annotator) and 630 for event 2 (210 per annotator). Table 1 and 2 presents the results for SET1 and SET2 when considering each individual annotator and all the annotators combined. The absolute error of $14.2 \mathrm{~ms}$ and $10.4 \mathrm{~ms}$ for SET1 and SET2 respectively when combining all the annotators compares favorably to absolute errors reported in the literature when adult data are annotated in a similar fashion.

Figure 5 shows that combining the annotations from the three experts resulted in a lower bias, a slope closer to one and higher goodness of fit $\left(\mathrm{R}^{2}=0.61\right)$ than any of the three annotators taken individually. The intraclass correlation coefficient (ICC) was also computed between each individual annotator annotations on the SQT and AQT to quantify how much the two sets of annotations resembled each other. ICC of 0.522 , $0.613,0.616$ for annotators $1-3$ were obtained. The relative ranking between the three annotators is in accordance with the $\chi^{2}$ evaluated (see Figure 5). 
Non-invasive fetal QT interval

Table 1: Individual annotators (A1-A3) and annotations for SET1 and SET2. Reference: FSE QT obtained from annotator A. Measure: non-invasive fetal ECG QT obtained from annotator A. RMSE95 and AE95:: RMSE and AE when removing the 5\% extreme values. All values are expressed in ms. The lowest absolute error (AE) is underlined and also corresponds to the lowest RMSE (root mean square error).

\begin{tabular}{ccccc}
\hline Method/Stats & RMSE & AE & RMSE95 & AE95 \\
\hline A1-EVENT1 & 27.5 & 22.5 & 25.0 & 20.7 \\
A2-EVENT1 & 41.3 & 32.8 & 35.9 & 29.5 \\
A3-EVENT1 & 21.6 & $\underline{17.1}$ & 19.2 & $\underline{15.5}$ \\
\hline A1-EVENT2 & 33.2 & 20.3 & 22.0 & 16.3 \\
A2-EVENT2 & 22.7 & 16.6 & 17.8 & 14.1 \\
A3-EVENT2 & 18.3 & $\underline{14.8}$ & 16.2 & $\underline{13.4}$ \\
\hline
\end{tabular}

Table 2: Combining cardiologists' annotations to get FSE QT and non-invasive fetal ECG QT for SET1 and SET2. The error is assessed for the mean/median/EM non-invasive fetal ECG QT against mean/median/EM FSE QT approaches for fusing the annotations. RMSE95 and AE95:: RMSE and AE when removing the 5\% extreme values. All values are expressed in ms. The lowest absolute error (AE) is underlined and also corresponds to the lowest RMSE (root mean square error).

\begin{tabular}{ccccc}
\hline Method/Stats & RMSE & AE & RMSE95 & AE95 \\
\hline Mean-EVENT1 & 17.9 & 14.1 & 15.1 & $\underline{12.4}$ \\
Median-EVENT1 & 21.3 & 17.1 & 18.7 & 15.5 \\
EM-EVENT1 & 18.0 & $\underline{14.2}$ & 15.3 & 12.7 \\
\hline Mean-EVENT2 & 15.4 & 11.5 & 12.1 & 9.9 \\
Median-EVENT2 & 18.8 & 14.2 & 15.8 & 12.5 \\
EM-EVENT2 & 13.6 & $\underline{10.4}$ & 11.4 & $\underline{9.2}$ \\
\hline
\end{tabular}



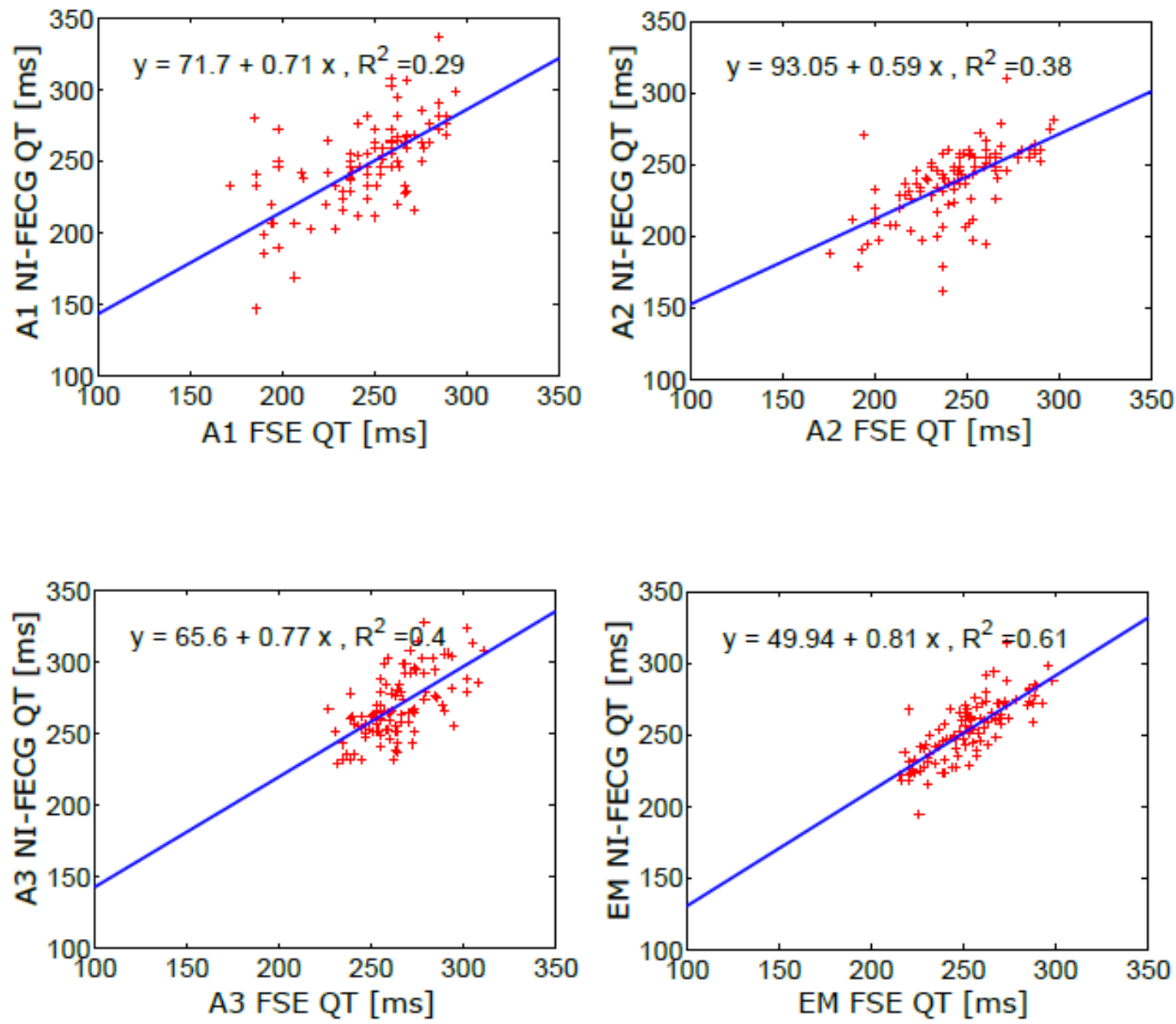

Figure 5: Plot of QT annotations from the extracted NI-FECG obtained using the NI-FECG monitor (denoted NI-FECG QT) against QT annotations from the FSE signal (denoted FSE QT), 22 fetuses (105, 1-min segments). A: annotator. (e.g. A1 NI-FECG QT refers to the QT annotated by annotator one on the NI-FEGC output from by the Meridian monitor). EM: crowd sourced annotations from the three clinicians using the EM algorithm (e.g. EM FSE QT refers to the scalp QT annotations merged using the EM algorithm). Line fit is given by: $\mathrm{y}=$ intercept + gradient $\mathrm{x}, \mathrm{R}^{2}$ is the corresponding coefficient of determination (goodness of fit). 
Non-invasive fetal QT interval

\section{Discussion and Conclusion}

This is the first paper to demonstrate that the fetal QT interval can be reliably measured from ECG data recorded non-invasively using electrodes on the maternal abdomen. Although we note that no pathologically long or short QT intervals were present in the data available, we do not see any significant reason to believe the signal processing of our FECG would lead to significant distortions, since we have shown in earlier work that low frequency components of the FECG are not distorted by our extraction process (Clifford et al., 2011). However, definitely proving this remains a topic for future studies with a significant prevalence of fetuses with short or long QT intervals.

Our annotators, who were blinded to the source of the waveform they were annotating, generated QT intervals with excellent correlation between abdominal data and corresponding FSE signal when averaged waveforms were used. In contrast, when individual waveforms were annotated, the distortion inherent to the waveforms led the annotators to generally identify shorter QT intervals when annotating the abdominal signals than the FSE signals (see figure 3). These findings, suggest that the most accurate approach to fetal QT annotation will be to use a waveform created from a running average of several heartbeats. This was confirmed by the quantitative analysis presented in Table 1 and 2 where the results for the experiment on SET2 were consistently better. Combining the annotations from the three electrophysiologists resulted in a lowering of the RMSE (from $18.3 \mathrm{~ms}$ to $13.6 \mathrm{~ms}$, SET2) and absolute error (14.8 ms to $10.4 \mathrm{~ms}$, SET2) compared to using any individual annotator. This is in accordance with the finding of Zhu et al. (2014) for adult QT annotation aggregation. In the case of the experiment on SET2 the expected maximization algorithm gave the best results.

The magnitude of the fetal QT estimation error obtained in this study (17.9 ms RMSE for SET1 and 13.6 ms RMSE for SET2) compare to the RMSE obtained when combining QT annotations from three annotators on adult ECG (RMSE of $16.07 \mathrm{~ms}$ found previously) (Zhu et al., 2014).

A number of published studies have attempted to extract the fetal QT (and other ECG morphology based quantities) from the NI-FECG or fetal magnetocardiography (Brambati and Pardi, 1980, Stinstra et al., 2002, Abboud et al., 1990, Taylor et al., 2005). However, these studies did not validate their measurements with invasive data and thus they did not prove that the algorithms that they used for NI-FECG extraction did not distorted the QT length, for example, through the distortion of the T-wave by heavy preprocessing of the abdominal data or by moving to the source domain using a blind source separation algorithm (Andreotti et al., 2016). 
Non-invasive fetal QT interval

Stinstra et al. (2002) used fetal magnetocardiography (MFCG) recordings from 582 healthy patients at different stages of the pregnancy (gestational age 17-41 weeks) and manually annotated the PR, PQ, QRS and QT intervals, averaging over 100 cardiac cycles per recording. They found the QT length was found to be in the interval [149 -339] $\mathrm{ms}(\mathrm{n}=412,16-42$ weeks of gestation), but did not have FSE data to validate their measurements. Brambati and Pardi (1980), used NI-FECG to record 421 pregnant women (17-41 weeks) performed a similar set of measurements, averaging 50 cardiac cycles per measurement, again without simultaneous measurement of invasive FECG data. Two other papers performed similar analyses and found the QT to range from 207-338 ms ( $\mathrm{n}=21,32-41$ weeks of gestation) (Abboud et al., 1990) and 233-329 ms ( $\mathrm{n}=11,24-41$ weeks of gestation) (Taylor et al., 2005). Although these studies did not validate their measurements with invasive data, the ranges found were similar to the ranges obtained in this paper (see figure $3)$.

It bears mentioning, however, that there is no gold standard for the fetal QT interval, given the inability to adhere standard electrodes to the fetal precordium. Validation using the FSE is a reasonable approach, however it would be useful to validate the fetal QT interval with ECG data measured immediately after birth. Such data would also provide information on whether the QT interval changes at delivery. Despite the fact that one of the principal advantages of the NI-FECG is its ability to perform antenatal monitoring, the study focused on measurements performed at birth. This is because this is the only alternative for obtaining a QT reference by using the FSE (other than using magnetocardiography, which is expensive and would prohibit the use of the NI-FECG monitor). However, it is important to mention that the accuracy in estimating the FQT from the NI-FECG will likely be lower if the gestational age was significantly lower, since the fetal heart would be smaller and the NI-FECG signal to noise ratio may therefore be lower.

Since the extraction and study of morphological parameters from the NI-FECG is a nascent field, it is difficult to say whether the error reported in this study is low enough to be considered acceptable for fetal QT monitoring. However, it is less than that quoted for adult ECG studies and thus demonstrates a promising application. In addition, it is important to note that recent attempts at estimating fetal QT automatically have provided a root mean square error of over $152 \mathrm{~ms}$, which indicates that our approach provides significant improvements (an order of magnitude reduction in errors) (Silva et al., 2013, Clifford et al., 2014).

Similar to prior studies (Silva et al., 2013), we determined that the QT interval can be most accurately measured by averaging a series of cardiac cycles. Averaging allows the production of quality ECG average cycles by reducing the signal to noise ratio by up to a factor $\sqrt{N}$ (where $N$ is the number of cycles averaged) 
Non-invasive fetal QT interval

under certain hypotheses (Rompelman and Ros, 1986b, Rompelman and Ros, 1986a). However this raises the question of how much 'averaging' should be allowed given that the ECG is a non-stationary signal. This question needs further investigation, together with the number of annotators and their associated skill levels needed to create an exact QT estimate (Zhu et al., 2014).

Despite its relatively small sample size, our study is unique in that we validated the non-invasive fetal ECG QT measurement for each subject against invasive data that is largely absent of potential artifact or error due to the automated extraction process, which is scientifically repeatable. In addition we presented a rigorous protocol for obtaining the fetal QT measurements using an online annotation interface designed by our group and by combining the medical annotations from three expert cardiologists. 
Non-invasive fetal QT interval

\section{Acknowledgment}

JB was supported by MindChild Medical Inc. North Andover, MA, by the UK Engineering and Physical

Sciences Research Council, the Balliol French Anderson scholarship fund, and by the Technion Aly Kaufman Fellowship.

\section{References}

ABBOUD, S., BARKAI, G., MASHIACH, S. \& SADEH, D. 1990. Quantification of the fetal electrocardiogram using averaging technique. Comput Biol Med, 20, 147-55.

AMER-WAHLIN, I., HELLSTEN, C., NOREN, H., HAGBERG, H., HERBST, A., KJELLMER, I., LILJA, H., LINDOFF, C., MANSSON, M., MARTENSSON, L., OLOFSSON, P., SUNDSTROM, A. \& MARSAL, K. 2001. Cardiotocography only versus cardiotocography plus ST analysis of fetal electrocardiogram for intrapartum fetal monitoring: a Swedish randomised controlled trial. Lancet, 358, 534-8.

AMERICAN COLLEGE OF OBSTETRICIANS AND GYNECOLOGISTS 2005. ACOG Practice Bulletin: Intrapartum fetal heart rate monitoring.

ANDREOTTI, F., BEHAR, J., ZAUNSEDER, S., OSTER, J. \& CLIFFORD, G. D. 2016. An open-source framework for stress-testing non-invasive foetal ECG extraction algorithms. Physiological Measurement, 37, 627.

BAZETT, H. C. 1920. An analysis of the time-relations of electrocardiograms. Heart, 7, 353-70.

BEHAR, J., ANDREOTTI, F., ZAUNSEDER, S., OSTER, J. \& CLIFFORD, G. D. 2016. A practical guide to noninvasive foetal electrocardiogram extraction and analysis. Physiological Measurement, 37, R1.

BEHAR, J., OSTER, J. \& CLIFFORD, G. D. 2014. Combining and benchmarking methods of foetal ECG extraction without maternal or scalp electrode data. Physiol Meas, 35, 1569-89.

BELFORT, M. A., SAADE, G. R., THOM, E., BLACKWELL, S. C., REDDY, U. M., THORP, J. M., JR., TITA, A. T., MILLER, R. S., PEACEMAN, A. M., MCKENNA, D. S., CHIEN, E. K., ROUSE, D. J., GIBBS, R. S., EL-SAYED, Y. Y., SOROKIN, Y., CARITIS, S. N., VANDORSTEN, J. P., EUNICE KENNEDY SHRIVER NATIONAL INSTITUTE OF CHILD, H. \& HUMAN DEVELOPMENT MATERNAL-FETAL MEDICINE UNITS, N. 2015. A Randomized Trial of Intrapartum Fetal ECG ST-Segment Analysis. N Engl J Med, 373, 632-41.

BRAMBATI, B. \& PARDI, G. 1980. The intraventricular conduction time of fetal heart in uncomplicated pregnancies. Br J Obstet Gynaecol, 87, 941-8.

CAMPBELL, R. W., GARDINER, P., AMOS, P. A., CHADWICK, D. \& JORDAN, R. S. 1985. Measurement of the QT interval. Eur Heart J, 6 Suppl D, 81-3.

CHRISTOV, I. \& SIMOVA, I. 2006. Fully automated method for QT interval measurement in ECG. Computers in Cardiology, 33, 321-324.

CLIFFORD, G., SAMENI, R., WARD, J., ROBINSON, J. \& WOLFBERG, A. J. 2011. Clinically accurate fetal ECG parameters acquired from maternal abdominal sensors. Am J Obstet Gynecol, 205, 47e1-35.

CLIFFORD, G. D., SILVA, I., BEHAR, J. \& MOODY, G. B. 2014. Non-invasive fetal ECG analysis. Physiol Meas, 35, 1521-36.

CROTTI, L., TESTER, D. J., WHITE, W. M., BARTOS, D. C., INSOLIA, R., BESANA, A., KUNIC, J. D., WILL, M. L., VELASCO, E. J., BAIR, J. J., GHIDONI, A., CETIN, I., VAN DYKE, D. L., WICK, M. J., BROST, B., DELISLE, B. P., FACCHINETTI, F., GEORGE, A. L., SCHWARTZ, P. J. \& ACKERMAN, M. J. 2013. Long QT syndromeassociated mutations in intrauterine fetal death. JAMA, 309, 1473-82.

DORET, M., MASSOUD, M., CONSTANS, A. \& GAUCHERAND, P. 2011. Use of peripartum ST analysis of fetal electrocardiogram without blood sampling: a large prospective cohort study. Eur J Obstet Gynecol Reprod Biol, 156, 35-40.

DUBNOV-RAZ, G., JUURLINK, D. N., FOGELMAN, R., MERLOB, P., ITO, S., KOREN, G. \& FINKELSTEIN, Y. 2008. Antenatal use of selective serotonin-reuptake inhibitors and QT interval prolongation in newborns. Pediatrics, 122, e710-5.

DUBNOV, G., FOGELMAN, R. \& MERLOB, P. 2005. Prolonged QT interval in an infant of a fluoxetine treated mother. Arch Dis Child, 90, 972-3.

FUJIMOTO, Y., MATSUMOTO, T., HONDA, N., TOJO, R., FURUYA, M., KASAI, K., SAITO, S., MOCHIMARU, F. \& ISHIKAWA, Y. 2009. Prenatal diagnosis of long QT syndrome by non-invasive fetal electrocardiography. J Obstet Gynaecol Res, 35, 555-61. 
Non-invasive fetal QT interval

GREENE, K. G. 1987. The ECG waveform. In: WHITTLE, M. (ed.) Baillieres clinical obstetrics and gynaecology. London: Bailliere Tindale.

GREENE, K. R. \& ROSEN, K. G. 1989. Long-term ST waveform changes in the ovine fetal electrocardiogram: the relationship to spontaneous labour and intrauterine death. Clin Phys Physiol Meas, 10 Suppl B, 33-40.

KESSLER, J., MOSTER, D. \& ALBRECHTSEN, S. 2013. Intrapartum monitoring of high-risk deliveries with ST analysis of the fetal electrocardiogram: an observational study of 6010 deliveries. Acta Obstet Gynecol Scand, 92, 75-84.

MOODY, G. B., KOCH, H. \& STEINHOFF, U. 2006. The physionet/computers in cardiology challenge 2006: QT interval measurement. Computers in Cardiology.

OUDIJK, M. A., KWEE, A., VISSER, G. H., BLAD, S., MEIJBOOM, E. J. \& ROSEN, K. G. 2004. The effects of intrapartum hypoxia on the fetal QT interval. BJOG, 111, 656-60.

ROMPELMAN, O. \& ROS, H. H. 1986a. Coherent averaging technique: a tutorial review. Part 1: Noise reduction and the equivalent filter. J Biomed Eng, 8, 24-9.

ROMPELMAN, O. \& ROS, H. H. 1986b. Coherent averaging technique: a tutorial review. Part 2: Trigger jitter, overlapping responses and non-periodic stimulation. J Biomed Eng, 8, 30-5.

SAMENI, R. \& CLIFFORD, G. D. 2010. A Review of Fetal ECG Signal Processing; Issues and Promising Directions. Open Pacing Electrophysiol Ther J, 3, 4-20.

SILVA, I., BEHAR, J., SAMENI, R., ZHU, T., OSTER, J., CLIFFORD, G. D. \& MOODY, G. B. Noninvasive fetal ecg: the physionet/computing in cardiology challenge 2013. Computing in Cardiology Conference (CinC), 2013, 2013. IEEE, 149-152.

STINSTRA, J., GOLBACH, E., VAN LEEUWEN, P., LANGE, S., MENENDEZ, T., MOSHAGE, W., SCHLEUSSNER, E., KAEHLER, C., HORIGOME, H., SHIGEMITSU, S. \& PETERS, M. J. 2002. Multicentre study of fetal cardiac time intervals using magnetocardiography. BJOG, 109, 1235-43.

TAYLOR, M. J., THOMAS, M. J., SMITH, M. J., OSEKU-AFFUL, S., FISK, N. M., GREEN, A. R., PATERSONBROWN, S. \& GARDINER, H. M. 2005. Non-invasive intrapartum fetal ECG: preliminary report. Bjog, $112,1016-21$.

WOLFBERG, A. J. \& NORWITZ, E. R. 2009. Probing the fetal cardiac signal for antecedents of brain injury. Clin Perinatol, 36, 673-84.

ZHU, T., JOHNSON, A. E., BEHAR, J. \& CLIFFORD, G. D. 2014. Crowd-sourced annotation of ecg signals using contextual information. Ann Biomed Eng, 42, 871-84. 